\title{
Optical circulator analysis and optimization: a mini-project for physical optics
}

\section{Zhujun Wan}

Zhujun Wan, "Optical circulator analysis and optimization: a mini-project for physical optics," Proc. SPIE 10452, 14th Conference on Education and

Training in Optics and Photonics: ETOP 2017, 104521I (16 August 2017); doi: 10.1117/12.2269669

SPIE Event: 14th Conference on Education and Training in Optics and Photonics, ETOP 2017, 2017, Hangzhou, China 


\title{
Optical circulator analysis and optimization: a mini-project for physical optics
}

\author{
Zhujun Wan* \\ Huazhong University of Science and Technology, 1037 Luoyu Rd., Wuhan, China
}

\begin{abstract}
One of the mini-projects for the course of physical optics is reported. The project is designed to increase comprehension on the basics and applications of polarized light and birefringent crystal. Firstly, the students are required to analyze the basic principle of an optical circulator based on birefringent crystal. Then, they need to consider the engineering optimization problems. The key tasks include analyzing the polarization transforming unit (composed of a halfwaveplate and a Faraday rotator) based on Jones matrix, maximizing the walk-off angle between e-ray and o-ray in birefringent crystal, separating e-ray and o-ray symmetrically, employment of a transformed Wollaston prism for input/output coupling of optical beams to fibers. Three years' practice shows that the project is of moderate difficulty, while it covers most of the related knowledge required for the course and helps to train the engineering thinking.
\end{abstract}

Keywords: Physical optics, optical circulator, birefringent crystal, polarized light, education

\section{INTRODUCTION}

As part of the education reform, we are trying to introduce mini-projects into the course of physical optics. The projects are designed for the students to increase comprehension on the theories and train the ability of analyzing real problems. This paper reported one of the mini-projects related to polarized light and crystal optics, which is an important part in the course of physical optics.

Optical circulator is a nonreciprocal device for optical fiber communication. Among the various approaches for an optical circulator, birefringent crystal is most popular in application. Based on the theories in text book, the students are required to analyze the principle of a crystal-based optical circulator and further optimize the device for engineering application.

The students build teams of 3-4 members and are required to accomplish a series of detailed tasks in their extracurricular team work. The key tasks include analyzing the polarization transforming unit (composed of a halfwaveplate and a Faraday rotator) based on Jones matrix, maximizing the walk-off angle between e-ray and o-ray in birefringent crystal for device minimization, separating e-ray and o-ray symmetrically for convenience of device assembly, employment of a transformed Wollaston prism for input/output coupling of optical beams to fibers. The project covers most of the related knowledge in polarized light and crystal optics.

\section{PRINCIPLE OF CRYSTAL-BASED OPTICAL CIRCULATOR}

\subsection{Operation of the optical circulator}

Optical circulator is a multi-port nonreciprocal device. Fig.1 shows the basic structure of a 3-port optical circulator based on birefringent crystal, which includes three beam displacers (BD) and four polarization transforming (PT) units. The PT unit is composed of a Faraday rotator (FR) and a $\lambda / 2$ waveplate (WP). When a linearly polarized optical beam passes through the PT unit forward (i.e. first the FR and then the $\lambda / 2 \mathrm{WP}$ ), the optical vector is rotated by $90^{\circ}$, while the optical vector remains unchanged when the beam passes backward (i.e. first the $\lambda / 2 \mathrm{WP}$ and then the FR).

Fig.1(a) and (b) trace the port (1) $\rightarrow$ (2) and port (2) $\rightarrow$ (3) light propagation respectively. The position of beam spot and orientation of optical vector at each plane are also presented. As shown in Fig.1 (a), a non-polarized optical beam inputs from port (1). The orthogonally polarized o-ray and e-ray are separated in BD1. Then one beam passes through PT unit 3 forward and the other beam passes through PT unit 2 backward. Thus the orthogonal optical vectors of the two beams become parallel before incidence on BD4. The two beams both act as o-ray in BD4 and pass it straightforward. Then one

14th Conference on Education and Training in Optics and Photonics: ETOP 2017, edited by Xu Liu,

Xi-Cheng Zhang, Proc. of SPIE Vol. 10452, 104521I - C 2017 ICO, IEEE, OSA, SPIE

CCC code: $0277-786 \mathrm{X} / 17 / \$ 18 \cdot$ doi: $10.1117 / 12.2269669$ 
beam passes through PT unit 6 backward and the other beam passes through PT unit 5 forward. The parallel optical vectors of the two beam become orthogonal before incidence on BD7. One acts as e-ray and the other acts as o-ray. The two beams are recombined by BD7 and output from port (2).

As shown in Fig.1 (b), a non-polarized optical beam inputs from port (2). The orthogonally polarized o-ray and e-ray are separated in BD7. Then one beam passes through PT unit 6 forward and the other beam passes through PT unit 5 backward. Thus the orthogonal optical vectors of the two beams become parallel before incidence on BD4. The two beams act as e-ray in BD4 and pass it with a lateral offset. Then one beam passes through PT unit 3 backward and the other beam passes through PT unit 2 forward. The parallel optical vectors of the two beam become orthogonal before incidence on BD1. One acts as o-ray and the other acts as e-ray. The two beams are recombined by BD1 and output from port (3).

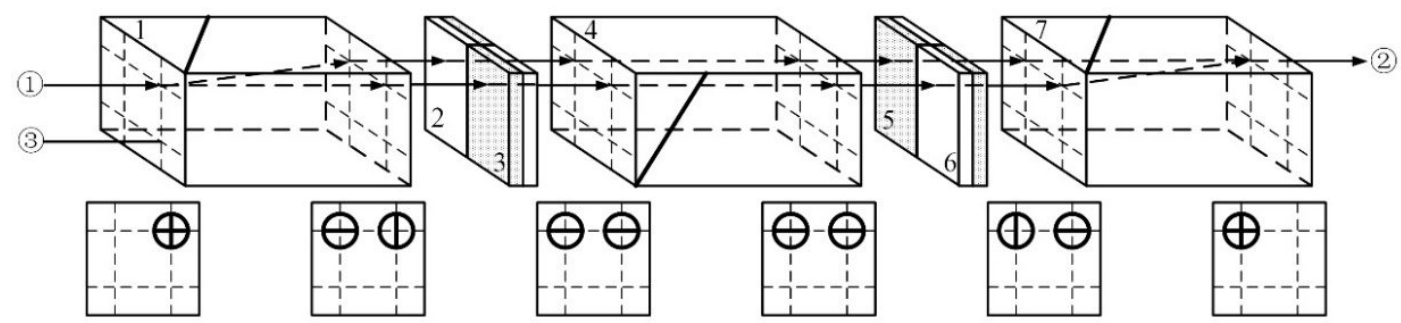

(a) Port (1) $\rightarrow$ (2)

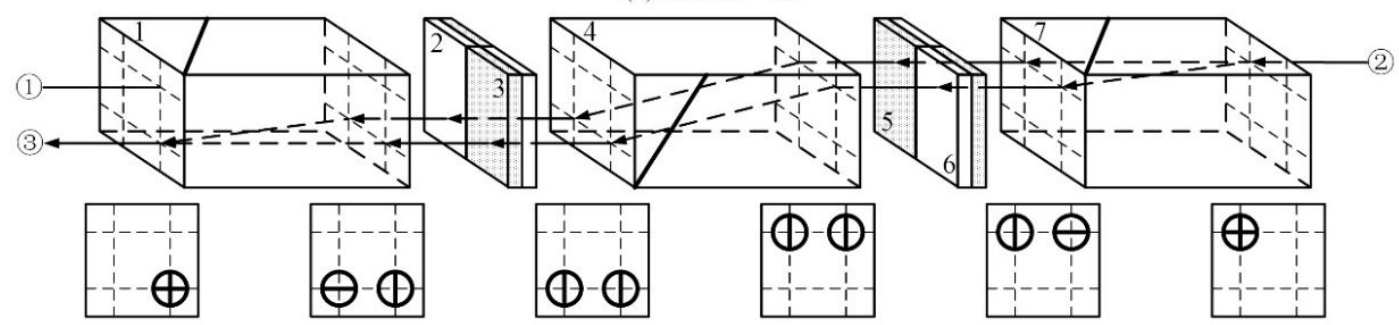

(b) Port (2) $\rightarrow$ (3)

Figure 1. Principle of the crystal-based optical circulator, 1,4,7 - beam displacer, 2,6 - $\lambda / 2$ waveplate + Faraday rotator, 3,5 - Faraday rotator $+\lambda / 2$ waveplate

Optical circulator is applied in optical fiber communication system. The input/output signals need to be coupled with optical fibers. In engineering applications, a dual-fiber collimator is placed between port (1) and (3) and a single-fiber collimator is placed at port (2). A dual-fiber collimator emits two collimated optical beams with a cross angle, while the two beams at port (1) and (3) are parallel, as shown in Fig.1. Thus a Wollaston prism is employed to deflect the two beams to match the dual-fiber collimator, as shown in Fig.2.

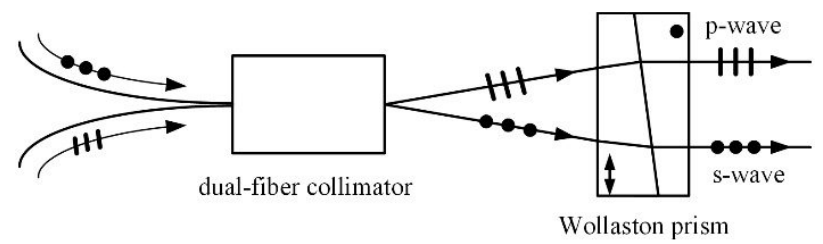

Figure 2. Matching between a dual-fiber collimator and a Wollaston prism

A conventional three-port optical circulator with consideration of coupling to input/output optical fibers is shown in Fig.3. A dual-fiber collimator, a single-fiber collimator and a Wollaston prism are added with respect to Fig.1. The Wollaston prism is placed between PT units 2,3 and BD4. 

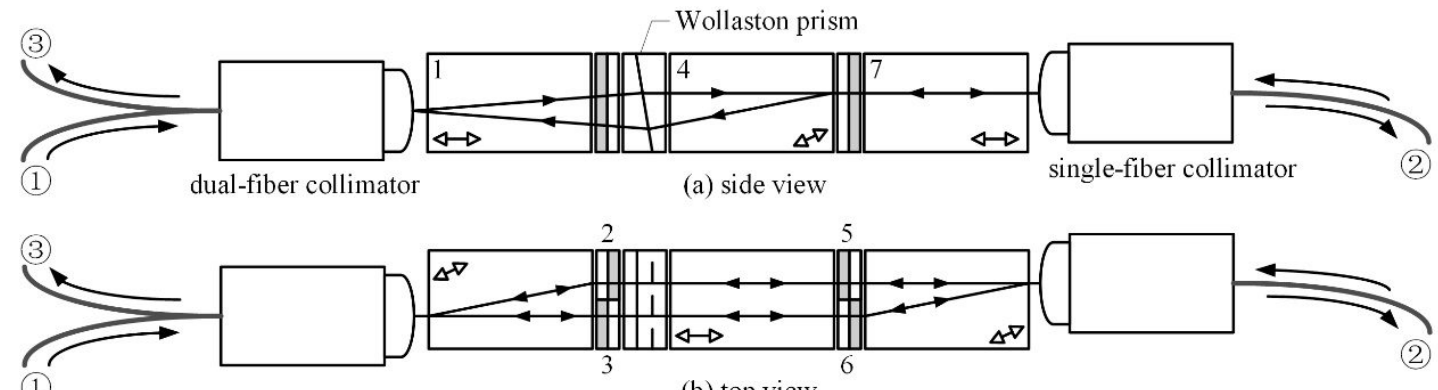

(1)

(b) top view

Figure 3. Structure of a conventional three-port optical circulator

It can be seen that the dual-fiber collimator and the single-fiber collimator are not coaxial in both side view and top view. This brings inconvenience for the device assembly. One of the key tasks of the project is to solve this problem.

\subsection{Principle of the key components}

The polarization transforming unit is one of the key components. The structure and polarization transforming function of a PT unit is shown in Fig.4. The FR rotates the optical vector by $45^{\circ}$ nonreciprocally, while the $\lambda / 2$ WP rotates the optical vector reciprocally. The optical axis of the $\lambda / 2 \mathrm{WP}$ is aligned as shown in Fig.4. Thus the PT unit rotates the optical vector by $90^{\circ}$ when the beam passes forward and the optical vector remains unchanged when the beam passes backward.

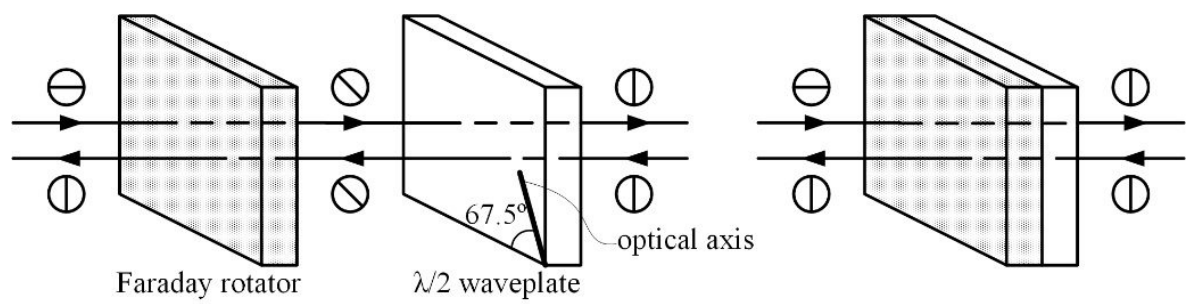

Figure 4. Structure and function of a polarization transforming unit

As another key component, the $\mathrm{BD}$ is made of birefringent crystal. The orthogonally polarized o-ray and e-ray are separated due to birefringence, as shown in Fig.5. The walk-off angle $\alpha$ depends on the birefringence coefficient $\Delta n=n_{0^{-}}$ $n_{e}\left(n_{o}\right.$ and $n_{e}$ are the refractive index of o-ray and e-ray respectively) and the cross angle $\theta$ between normal of e-wave (same as o-ray in Fig.5) and optical axis of the crystal, as shown in Eq. (1) [1].

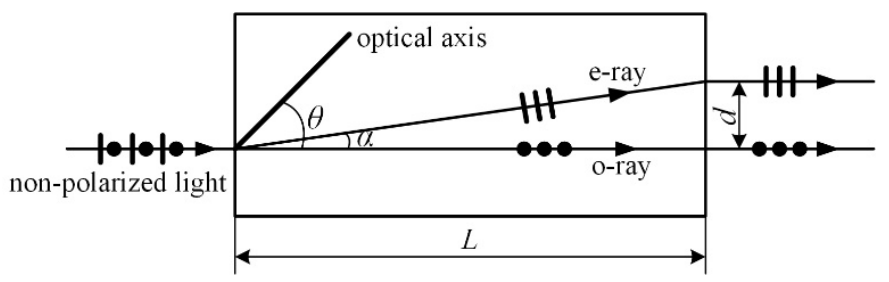

Figure 5. Structure of the beam displacer 


$$
\tan \gamma=\left(1-\frac{n_{o}^{2}}{n_{e}^{2}}\right) \frac{\tan \theta}{1+\frac{n_{o}^{2}}{n_{e}^{2}} \tan ^{2} \theta}
$$

\section{KEY TASKS OF THE PROJECT}

Based on above principles of the crystal-based optical circulator and the key components, the students are required to accomplish the following key tasks. The textbook theories related to polarized light and crystal optics are employed to analyze the real problems and the engineering consideration for application of the device is required.

\subsection{Mathematics of the polarization transforming unit}

According to the theories in polarized light and crystal optics, the polarized light can be mathematically characterized by a Jones vector and the function of a crystal component can be characterized by a Jones matrix. Thus when a polarized light passes through a series of crystal components, the polarization state of the output light can be mathematically derived.

The PT unit consists of two crystal components. The students are required to analyze the function of the PT unit mathematically. The Jones matrices of the $\lambda / 2 \mathrm{WP}$ and the FR are as Eqs. (2) and (3). The Jones vector of a linear polarized light is as Eq. (4) [2].

$$
\begin{aligned}
& G_{1}=\left[\begin{array}{cc}
\cos 2 \beta & \sin 2 \beta \\
\sin 2 \beta & -\cos 2 \beta
\end{array}\right]=\frac{1}{\sqrt{2}}\left[\begin{array}{cc}
-1 & 1 \\
1 & 1
\end{array}\right] \\
& G_{2}=\left[\begin{array}{cc}
\cos \gamma & -\sin \gamma \\
\sin \gamma & \cos \gamma
\end{array}\right]=\frac{1}{\sqrt{2}}\left[\begin{array}{cc}
1 & -1 \\
1 & 1
\end{array}\right] \\
& E_{i}=\left[\begin{array}{l}
\cos \phi \\
\sin \phi
\end{array}\right]
\end{aligned}
$$

where the axis orientation of the $\lambda / 2 \mathrm{WP}$ is $\beta=67.5^{\circ}$ and the rotation angle by the FR is $\gamma=45^{\circ}$.

When the linear polarized light passes through the PT unit forward and backward, the polarizations of the output light are as Eqs. (5) and (6).

$$
\begin{aligned}
& E_{o f}=G_{1} G_{2} E_{i}=\left[\begin{array}{c}
\cos \left(\phi-90^{\circ}\right) \\
\sin \left(\phi-90^{\circ}\right)
\end{array}\right] \\
& E_{o b}=G_{2} G_{1} E_{i}=\left[\begin{array}{l}
\cos \left(180^{\circ}-\phi\right) \\
\sin \left(180^{\circ}-\phi\right)
\end{array}\right]
\end{aligned}
$$

Eq. (5) means that the optical vector of the output light is rotated by $90^{\circ}$ when the light passes forward, while Eq. (6) means that the optical vector remains unchanged when the light passes backward given $\phi=0^{\circ}$ or $90^{\circ}$.

\subsection{Optimization of the beam displacer}

In a BD crystal, the walk-off angle $\alpha$ between e-ray and o-ray is as Eq. (1). Maximally separating the two rays with a minimal crystal length will help to minimize the optical circulator. It can be derived from Eq. (1) that the maximal walkoff angle $\alpha_{\max }$ is as Eq. (7) when the optical axis orientation $\theta_{c}$ of the crystal is as Eq. (8). For the mostly used crystal $\mathrm{YVO}_{4}$, the refractive indices are $n_{o}=1.9447$ and $n_{e}=2.1486$ at wavelength $\lambda=1550 \mathrm{~nm}$, the maximum walk-off angle is $\alpha_{\max }=5.7^{\circ}$. Thus the ratio between the crystal length and the beam walk-off is $L: d=10: 1$. 


$$
\begin{aligned}
& \alpha_{\max }=\arctan \left[\frac{1}{2}\left(\frac{n_{e}}{n_{o}}-\frac{n_{o}}{n_{e}}\right)\right] \\
& \theta_{c}=\arctan \left(\frac{n_{e}}{n_{o}}\right)
\end{aligned}
$$

As we can see in a conventional BD as Fig.5, the e-ray and o-ray are not symmetrically separated referring to the input light. Thus ports (1) and (3) are not symmetric referring to port (2) in the vertical plane, as shown in Fig.1. A modified BD is designed as Fig.6. $S_{o}$ and $S_{e}$ indicate o-ray and e-ray, $K_{o}$ and $K_{e}$ indicate wave normal of the ordinary and extraordinary lightwave. The surface is polished by a slant angle $\delta$ and the optical axis keeps cross angle $\theta_{c}$ referring to $K_{e}$. Thus e-ray and o-ray are maximally and symmetrically separated with walk-off angle $\alpha_{\max }$. The students are required to design the modified $\mathrm{BD}$ based on solving of a transcendental equation and tracing of both o-ray and eray.

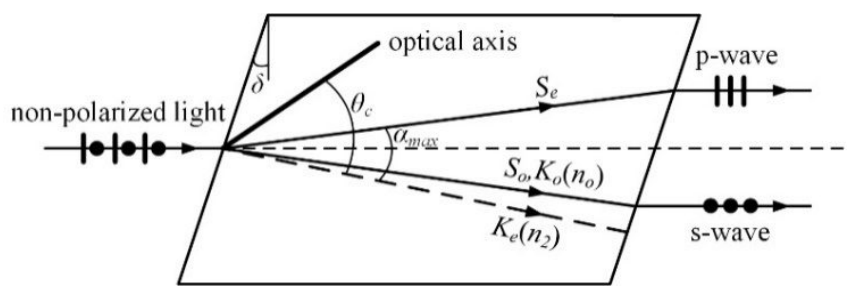

Figure 6. Structure of the modified beam displacer

With the modified BD employed in the optical circulator, the input/output ports become coaxial in the top view, as shown in Fig.7.

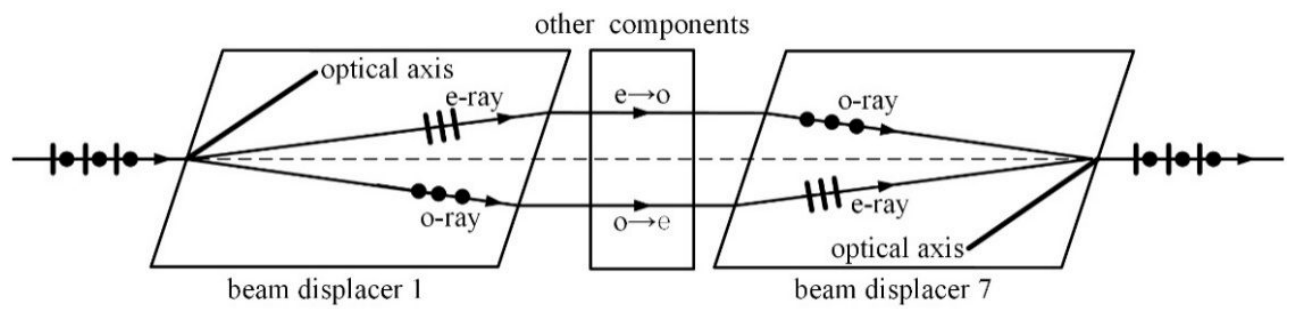

Figure 7. Employment of the modified beam displacer in the optical circulator

\subsection{Design of a transformed Wollaston prism}

As shown in Fig.3(a), the Wollaston prism is placed before BD4. The cooperation of the Wollaston prism and BD4 is shown in Fig.8. The two polarized beams (p-wave and s-wave) are not symmetric with respect to the non-polarized light, which is the cause resulting in asymmetry in the top view of Fig.3.

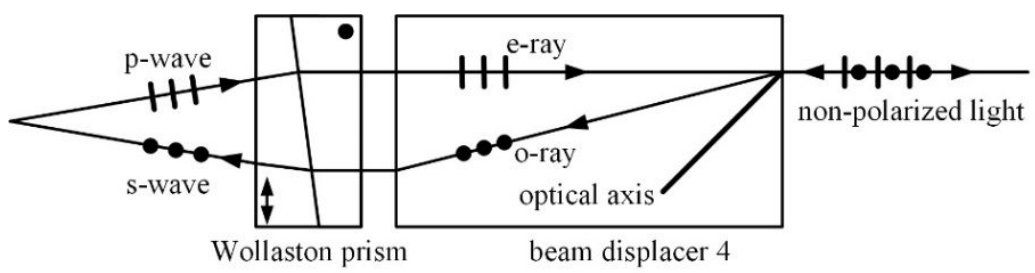

Figure 8. Combination of the Wollaston prism and beam displacer 4 
A transformed Wollaston prism is designed as Fig.9. It consists of two birefringent wedges. The optical axes of the two wedges are orthogonal and o-ray and e-ray exchange in the two wedges, which is the characterization of a Wollaston prism. However, the optical axis of wedge 2 is not parallel to the surface. There is a walk-off between e-ray and o-ray in wedge 2. It shows the characterization of a $\mathrm{BD}$. Thus the transformed Wollaston prism shows both functions of a Wollaston prism and a BD. It can replace the combination of the Wollaston prism and BD4 shown in Fig.8.

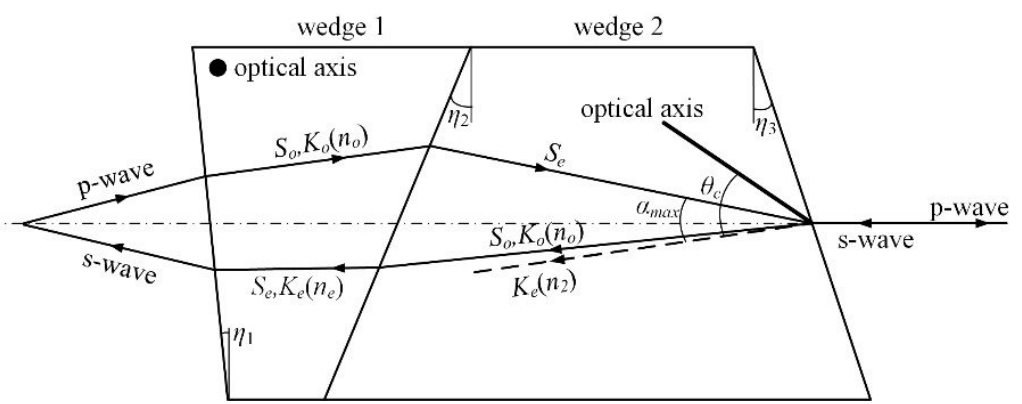

Figure 9. Structure of the transformed Wollaston prism

The students are required to design the transformed Wollaston prism. The cross angle between p-wave and s-wave on the left must match the dual-fiber collimator in Fig.2 and the two waves need to be symmetric with respect to the combined p-wave and s-wave on the right. In order to minimize the thickness of the wedges, the e-ray and o-ray in wedge 2 is to be maximally separated. The optional parameters include slant angles $\eta_{1}, \eta_{2}, \eta_{3}$ of the three surfaces, the thickness of the two wedges and the optical axis orientation of wedge 2. All these can be accomplished based on solving of a transcendental equation and tracing of both o-ray and e-ray in the wedges.

With the modified BD1 and BD4, and by replacing the conventional Wollaston prism and BD4 with a transformed Wollaston prism, the structure of the optimized three-port optical circulator is as Fig.10. It can be seen that the dual-fiber collimator and the single-fiber collimator is coaxial in both the side view and the top view. Minimization and coaxial assembly of the device is accomplished.

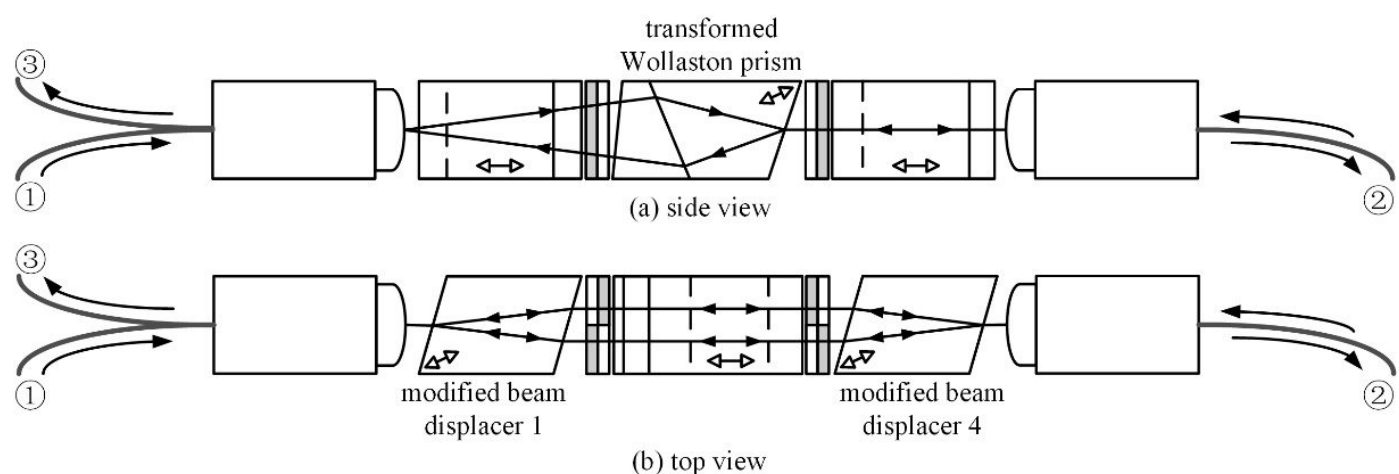

Figure 10. Structure of the optimized three-port optical circulator

\section{CONCLUSION}

The mini-project is designed for the course of physical optics and related to the chapter of polarized light and crystal optics. The students are required to analysis a crystal-based optical circulator based on the textbook theories. The key tasks are aimed to optimize of the device for engineering application and cover most of the knowledge in the chapter. 
Three years' practice shows that the project is of moderate difficulty for the undergraduates. Most of the students accomplish the tasks well based on team work.

\section{REFERENCES}

[1] Maria C. Simon, Karin V. Gottschalk, "Waves and rays in uniaxial birefringent crystals," Optik 118, pp. 457-470 (2007)

[2] Yang Zhang, Changxi Yang, Shiguang Li, He Yan, Jingchan Yin, Claire Gu, and Guofan Jin, "Complete polarization controller based on magneto-optic crystals and fixed quarter wave plates," Optics Express 14(8), pp. 3484-3490 (2006) 